\section{Tabagismo associado a outros fatores comportamentais de risco de doenças e agravos crônicos não transmissíveis}

\author{
Smoking associated with other behavioral risk \\ factors for chronic non-communicable diseases
}

\author{
${ }^{1}$ Faculdade de Medicina \\ de Botucatu, Universidade \\ Estadual Paulista, Botucatu, \\ Brasil. \\ 2 Universidade Federal do \\ Pará, Belém, Brasil. \\ Correspondência \\ S. J. P. Berto \\ Faculdade de Medicina de \\ Botucatu, Universidade \\ Estadual Paulista. \\ Distrito de Rubião Júnior $s / n$, \\ Botucatu, SP \\ 18618-000, Brasil. \\ spberto@fmb.unesp.br
}

\begin{abstract}
The study interviewed 1,410 adults by telephone. Respondents comprised a random sample and represented the population over 18 years of age living in households with landline telephone services. Smoking prevalence was $21.8 \%$, higher in males (25\%) and in the 18-29 year bracket. Smoking and sedentary lifestyle occurred together in $13.9 \%$ of males and $14.2 \%$ of females; smoking and low fruit consumption in $12.9 \%$ of males and $12.3 \%$ of females; and smoking and low vegetable consumption in 5.8\% of males and 5.1\% of females. An association between smoking and excessive alcohol intake was only observed in males (3.5\%). As observed for smoking alone, the simultaneous occurrence of smoking and other behavioral risk factors for CNCD was inversely associated with schooling. Evidence of clustering between smoking and sedentary lifestyle, smoking and excessive alcohol intake, and smoking and improper diet thus calls for interventions focused on prevention and the concomitant reduction of major behavioral risk factors.
\end{abstract}

Smoking; Sedentary Lifestyle; Life Style; Food Habits
Silvia Justina Papini Berto 1

Maria Antonieta Barros Leite Carvalhaes 1 Erly Catarina de Moura ${ }^{2}$

\section{Introdução}

Estimativas da Organização Mundial da Saúde (OMS) apontam que as doenças e agravos crônicos não transmissíveis (DANT) são responsáveis por $58,5 \%$ de todas as mortes e por $45,9 \%$ da carga total global de doenças expressa por anos perdidos de vida saudável. No Brasil, mortes por doenças respiratórias, cardiovasculares, câncer e diabetes, entre outras, somaram $62,8 \%$ das mortes documentadas em 2004. A projeção da OMS é que esse grupo de doenças seja a primeira causa de morte em todos os países em desenvolvimento até 2010. Tabagismo, consumo abusivo de bebidas alcoólicas, obesidade, consumo excessivo de gorduras saturadas, ingestão insuficiente de frutas e hortaliças e inatividade física são os principais fatores de risco modificáveis responsáveis pela maioria das mortes por DANT e por fração substancial da carga de doenças em razão dessas enfermidades, de acordo com a OMS 1.

Estudos mostram que esses fatores tendem a ocorrer simultaneamente 2,3,4,5 e que a presença concomitante de tabagismo e outros fatores de risco para DANT eleva a probabilidade do indivíduo desenvolver doenças crônicas (doença cardiovascular, câncer etc.) além do aumento que corresponderia à soma dos riscos associados a cada fator separadamente, agravando significativamente o risco de mortalidade 6,7.

Em Madri, Espanha, cerca de 20\% dos adultos apresentavam três ou quatro fatores de risco 
simultâneos, sendo tabagismo o fator que apareceu em mais associações 4 . Em uma população composta de 1.465 indivíduos adultos de Bogotá, Colômbia, o sedentarismo foi o fator mais comumente associado a outros, pois $42 \%$ dos indivíduos apresentavam dois e $21 \%$ três fatores de risco simultâneos 5. Tabagismo em associação com consumo abusivo de álcool tem sido amplamente detectado em estudos internacionais 4,8,9,10.

Dos fatores comportamentais de risco implicados na gênese de muitas doenças crônicas, o tabagismo é um dos principais, sendo a principal causa de morte evitável em todo o mundo atualmente 4 milhões de óbitos anuais, podendo chegar, em 2030, a 10 milhões de mortes 1 . Além da mortalidade, o hábito de fumar está associado ao desenvolvimento de hipertensão, aterosclerose, infarto do miocárdio, acidente vascular cerebral, enfisema pulmonar, doenças respiratórias, coronariopatias e vários tipos de câncer (pulmão, boca, laringe, próstata e outros) 11 .

No estudo de Madri, já citado, além das altas taxas de tabagismo $(41,7 \%$ nos homens e $36,6 \%$ nas mulheres), o resultado considerado mais relevante pelos autores foi identificar que apenas $3,1 \%$ e $3,4 \%$ de homens e mulheres, respectivamente, eram somente tabagistas, isto é, não apresentavam simultaneamente outro fator de risco de DANT. Cerca de 19\% dos homens apresentavam tabagismo e mais um segundo fator e $15,4 \%$ tabagismo e dois outros fatores. Nas mulheres, $20,1 \%$ eram tabagistas e apresentavam também um segundo fator, $11,4 \%$, além de tabagistas, apresentavam mais outros dois fatores de risco de DANT 4.

No Brasil, estudo com idosos investigou a presença simultânea de dois ou mais fatores de risco de DANT (incluindo fatores comportamentais e não comportamentais), detectando-a em $71,3 \%$ dos indivíduos 9 . Na Região Sul do Brasil, um estudo populacional detectou que $53,4 \%$ da população adulta apresentavam dois ou mais fatores de risco de doenças crônicas 12 e em Salvador, Bahia $13,69 \%$ dos homens e $68,1 \%$ das mulheres adultas apresentavam dois ou mais fatores de risco (comportamentais e/ou bioquímicos) de DANT 13. Embora possíveis de serem produzidas a partir dos dados do sistema VIGITEL 14, estimativas da ocorrência simultânea de fatores de risco de DANT na população adulta brasileira ainda não estão disponíveis.

Considerando os estudos brasileiros acima citados e os realizados em outros países 4,5,10, parece de particular interesse o conhecimento sobre a ocorrência simultânea de tabagismo e outros fatores comportamentais em contextos específicos, tanto para orientar a política de pro- moção da saúde como, eventualmente, ampliar o foco dos programas de cessação deste hábito.

$\mathrm{O}$ presente artigo apresenta a prevalência de tabagismo e a prevalência simultânea de tabagismo e outros fatores comportamentais de risco de DANT (sedentarismo, consumo excessivo de álcool, obesidade e alimentação inadequada) em adultos a partir de dados obtidos com a aplicação do sistema VIGITEL, no Município de Botucatu, São Paulo, no período de 2004/2005 15.

\section{Método}

Delineamento

Os dados analisados são provenientes de um estudo transversal, mediante entrevistas telefônicas, realizado entre 2004/2005 no Município de Botucatu, que constituiu a linha de base de um sistema de monitoramento de fatores de risco de DANT modificáveis por intervenções de saúde. A concepção do estudo, o processo de amostragem, o questionário e a coleta de dados foram descritos detalhadamente em publicações anteriores $14,15,16$ e são apresentados resumidamente, a seguir.

\section{Amostra}

Foi calculado em 1.000 o número mínimo de entrevistas a serem realizadas para poder estimar, com coeficiente de confiança de pelo menos $95 \%$ e erro máximo de $5 \%$, a freqüência de consumo de tabaco e de outros fatores de risco de DANT na população adulta do Município de Botucatu.

Devido à dificuldade de se estimar o número de linhas que deveriam ser sorteadas para a obtenção do número desejado de linhas elegíveis (linhas residenciais ativas), o processo de amostragem foi realizado em etapas:

i) Inicialmente, foram sorteadas 1.867 linhas do cadastro da empresa responsável pela telefonia fixa do Município do Botucatu. O sorteio foi realizado por funcionário da empresa, com acompanhamento de um membro da equipe responsável pelo projeto;

ii) A seguir, essas linhas foram re-sorteadas e divididas em seis réplicas de 311/312 linhas, de modo que cada réplica contivesse uma subamostra representativa da total;

iii) Apenas após a confirmação da elegibilidade (linha residencial ativa) de cada linha, realizouse o sorteio de um morador adulto (18 anos ou mais) por linha. Este sorteio foi feito com base em seqüências aleatórias de números. A entrevista era então agendada ou realizada imediata- 
mente, se a pessoa sorteada estivesse presente e disponível.

Do total de linhas sorteadas, $1.622(86,9 \%)$ foram confirmadas como elegíveis, sendo número fora de serviço a principal causa de não elegibilidade $(\mathrm{n}=136)$. Entretanto, a amostra estudada consistiu de 1.410 adultos, e as perdas (212) em relação às elegíveis decorreram de vários motivos: 36 linhas não puderam ser contatadas após 10 tentativas, em dias de semana e horários variados, não tendo sido possível sortear o indivíduo a ser entrevistado; 95 indivíduos sorteados se recusaram a participar do estudo; 60 não foram mais contatados após terem concordado e agendado a entrevista. Houve ainda 21 indivíduos que iniciaram a entrevista, mas a interromperam, solicitando contato futuro que não pôde ser realizado. Assim, a taxa de sucesso do sistema foi de $86,9 \%$ (proporção de entrevistados sobre o total de linhas elegíveis), havendo $5,8 \%(n=95)$ de recusas e $7,3 \%$ de perdas por outras razões.

\section{Questionário e coleta de dados}

O questionário foi construído de modo a permitir entrevistas telefônicas com o emprego de computadores, ou seja, as perguntas eram lidas diretamente da tela do monitor de vídeo e as respostas registradas diretamente em meio eletrônico, o que permitia viabilizar a alimentação direta e contínua de banco de dados em formato "dbase”. Incluiu 74 questões sobre características demográficas e sócio-econômicas dos entrevistados, padrões de alimentação e de atividade física, consumo de cigarros e de bebidas alcoólicas, peso e altura referidos pelos entrevistados e referência a diagnóstico médico anterior de hipertensão arterial e diabetes. Para este artigo foram usadas as questões sobre consumo de tabaco: consumo atual de tabaco; consumo de tabaco no passado; número de cigarros consumidos por dia e, também, idade, sexo, sedentarismo, estado nutricional, escolaridade, freqüência de consumo de bebida alcoólica, vegetais e frutas.

A coleta de dados ocorreu no período de 20 de setembro a 20 de dezembro de 2004 e entre 15 a 30 de janeiro de 2005, envolvendo equipe técnica composta por três entrevistadores e um supervisor.

\section{Variáveis em estudo}

As variáveis utilizadas neste estudo foram:

i) Tabagismo: para descrever o tabagismo na população foram considerados os seguintes indicadores: consumo atual de tabaco (sim, não); con- sumo de tabaco no passado (sim, não); número de cigarros consumidos por dia $(<10,10-19$, $20 \mathrm{e}+$ ).

ii) Sedentarismo: foi definido como a proporção de indivíduos que não praticava qualquer atividade física de lazer nos três meses anteriores, que não realizava esforços intensos no trabalho, que não se deslocava para o trabalho caminhando ou de bicicleta e que não era responsável pela "limpeza pesada" de suas casas.

iii) Baixo consumo de frutas: situação atribuída aos indivíduos que referiram não ingerir regularmente este grupo de alimentos em 5 ou mais dias da semana.

iv) Baixo consumo de saladas: situação atribuída aos indivíduos que referiram não ingerir regularmente este grupo de alimentos em 5 ou mais dias da semana.

v) Baixo consumo de alimentos reguladores: situação atribuída aos indivíduos que não consumiam regularmente (em 5 ou mais dias da semana) frutas, saladas e legumes.

vi) Consumo excessivo de bebidas alcoólicas: foi considerado consumo excessivo quando o entrevistado relatou consumir em 5 ou mais dias da semana mais de duas doses de bebida, para homens, e mais de uma dose, para mulheres.

vii) Estado nutricional: com base no IMC, categorizado em sem excesso de peso (IMC $<25 \mathrm{~kg} / \mathrm{m}^{2}$ ), com excesso de peso (IMC $\geq 25 \mathrm{~kg} / \mathrm{m}^{2}$ ) e obesos (IMC $\geq 30 \mathrm{~kg} / \mathrm{m}^{2}$ ).

viii) Escolaridade: em 3 níveis, 0-8, 9-11 e $\geq 12$ anos.

ix) Faixa etária: em 5 níveis, 18-29, 30-39, 40-49, 50-59 e 60 ou mais anos de idade.

\section{Análise dos dados}

Para produzir estimativas válidas para o conjunto da população adulta do município, foram adotados dois fatores de ponderação. Foram calculadas inicialmente estimativas da prevalência dos indicadores para a população adulta com telefone do Município de Botucatu e, neste caso, foi empregado fator individual de ponderação, correspondente ao número de adultos no domicílio do entrevistado multiplicado pelo inverso do número de linhas telefônicas. Para corrigir a eventual sub ou super-representação de determinados estratos demográficos e sócio-econômicos nesta população, com relação à população adulta total do município, adotou-se um segundo fator adicional de ponderação, que veio a ser a razão entre a freqüência relativa de indivíduos presentes nesses estratos na população estudada pelo VIGITEL e na população adulta total do município estudada pelo Censo Demográfico de 2000 17. Maiores detalhes do cálculo desse se- 
gundo fator de ponderação podem ser obtidos consultando-se publicações anteriores 14,15.

O processamento de dados e as análises estatísticas foram feitos com o auxílio do programa SPSS versão 13.0 (SPSS Inc., Chicago, Estados Unidos), que permite o uso de amostras ponderadas. Para avaliar a significância estatística das associações pesquisadas, empregou-se teste estatístico baseado na distribuição do qui-quadrado, (com $\mathrm{p}<0,05$ como nível crítico). Para verificar a tendência, usou-se o teste 2 de tendência pela estatística de Cochran-Armitage (programa SAS, v 9.1; SAS Inst., Cary, Estados Unidos).

\section{Aspectos éticos}

Conforme adotado na primeira aplicação pioneira do sistema VIGITEL 16, no Município de São Paulo, tratando-se de uma pesquisa por telefone, o consentimento livre e esclarecido assinado foi substituído pelo consentimento verbal, obtido por ocasião dos contatos telefônicos com os entrevistados. Esclareceu-se que os dados obtidos seriam utilizados apenas para fins de pesquisa e monitoramento de fatores de risco para DANT, subsidiando o desenvolvimento de intervenções locais. A todos os entrevistados forneceu-se um número de telefone para esclarecimento de dúvidas quanto ao projeto. Este estudo foi aprovado pelo Comitê de Ética em Pesquisa da Faculdade de Saúde Pública da Universidade de São Paulo e contou também com a ciência e aprovação do Comitê de Ética em Pesquisa da Faculdade de Medicina de Botucatu da Universidade Estadual Paulista.

\section{Resultados}

A Figura 1 apresenta a distribuição dos homens e mulheres segundo o consumo de tabaco atual ou passado. Em conjunto, a maioria nunca fumou, porém a freqüência de fumantes e ex-fumantes (53\%) em homens superou a daqueles que nunca fumaram. Entre fumantes, cerca de $40 \%$ fumam 20 ou mais cigarros ao dia e apenas $21 \%$ menos de 10, mas homens e mulheres têm comportamentos diferentes com relação ao número de cigarros consumidos: $15 \%$ e $28 \%$, respectivamente, fumam menos de 10 cigarros por dia. Tais dados constam da Figura 2.

A Tabela 1 apresenta as taxas de tabagismo em homens e mulheres segundo idade, escolaridade e estado nutricional. Observou-se uma associação não linear entre tabagismo e idade. As taxas mais altas ocorreram nos jovens (18-29 anos) e também nos adultos entre 40-49 anos, as menores, entre 30-39 e acima de 60 anos. Constatou-se queda abrupta da prevalência na faixa etária entre 30 e 39 anos em comparação à observada nos mais jovens, e queda progressiva do tabagismo a partir de 40 anos. Em relação à escolaridade, encontrou-se associação linear e inversa: queda da prevalência de tabagismo com aumento do nível de escolaridade, tanto para os homens quanto para mulheres. A associação entre tabagismo e estado nutricional variou segundo sexo. Em homens, as taxas de fumantes caem de $33,2 \%$ em indivíduos sem excesso de peso para $24 \%$ naqueles com sobrepeso e $4 \%$ nos obesos, comportamento distinto do observado nas mulheres: menores freqüências naquelas com sobrepeso e prevalências semelhantes no grupo sem excesso de peso e em obesas.

$\mathrm{Na}$ Tabela 2, apresentam-se as prevalências de tabagismo associado a outros fatores de risco comportamentais em homens e mulheres adultos do Município de Botucatu. A associação mais freqüente foi tabagismo e sedentarismo, presente em $13,9 \%$ dos homens e $14,2 \%$ das mulheres do município; em seguida, com freqüências um pouco menores, tabagismo e baixo consumo de frutas. A prevalência de tabagismo e consumo excessivo de álcool foi de 3,5\% nos homens, associação de fatores não observada nas mulheres.

A escolaridade se associou com a prevalência simultânea de tabagismo e outros fatores de risco de DANT, dados que podem ser observados na Tabela 3. Em homens, a prevalência de tabagismo e consumo excessivo de álcool cai com o aumento da escolaridade. A mesma tendência foi observada, em homens e mulheres, para tabagismo junto com sedentarismo e para tabagismo com excesso de peso.

\section{Discussão}

A validade de estimativas de prevalências de fatores de risco de doenças e agravos crônicos não transmissíveis obtidas por meio do inquérito telefônico VIGITEL tem sido demonstrada 18,19. Destaca-se que a pesquisa em Botucatu apresentou elevada proporção de entrevistas completadas em relação ao total de indivíduos sorteados $(86,9 \%)$, ligeiramente superior à observada em São Paulo $(84,7 \%)$ 15. Não sendo universal a cobertura domiciliar de tefonia fixa, uma limitação dos dados é a menor proporção de indivíduos do sexo masculino, de idades menores de 39 anos e dos menores estratos de escolaridade na amostra em relação à população total do município. Entretanto, com a aplicação de fatores de ponderação, as estimativas foram ajustadas para essas diferenças, e se pode considerá-las válidas para adultos do Município de Botucatu 15. 
Distribuição de homens, mulheres e população total segundo situação atual em relação ao tabagismo. Botucatu, São Paulo, Brasil, 2004
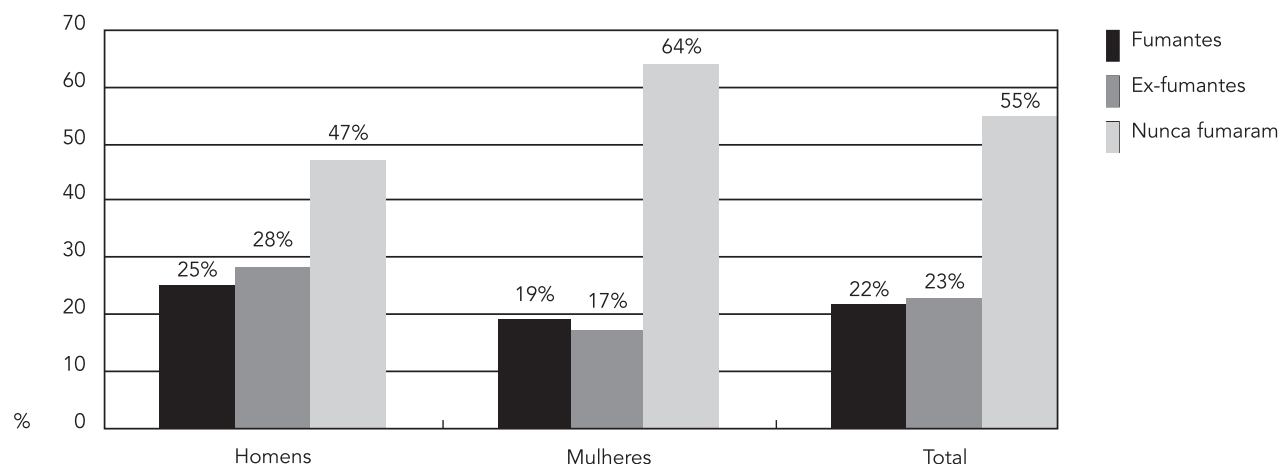

$\prod$ Nunca fumaram

Figura 2

Distribuição dos tabagistas homens e mulheres segundo situação e número habitual de cigarros consumidos por dia. Botucatu, São Paulo, Brasil, 2004.

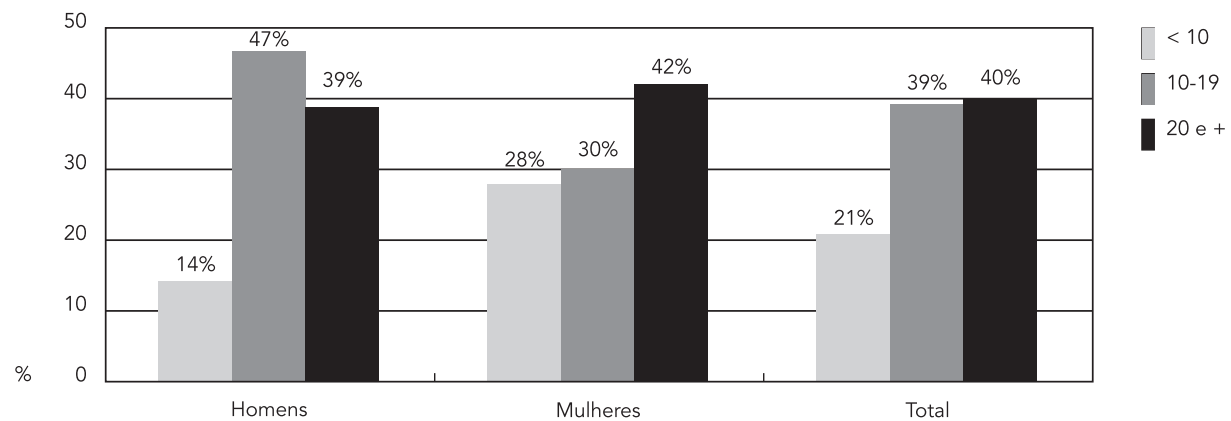

A prevalência de tabagismo $(21,8 \%)$ foi semelhante à obtida em São Paulo (21\%), em 2008, ano em que esta cidade foi a capital com maior prevalência de fumantes no Brasil. Destaca-se também que $8,4 \%$ dos indivíduos adultos de Botucatu eram tabagistas pesados ( $>20$ unidades/dia), índice bastante alto se comparado aos obtidos para as capitais brasileiras pelo VIGITEL em 2008 14, quando a maior frequência de fumantes pesados foi $8,2 \%$, em Porto Alegre. Esses resultados situam Botucatu no grupo de municípios brasileiros com altas taxas de tabagismo e apontam a necessidade de intervenções, tanto para prevenção do início de tabagismo em jovens como para a cessação deste hábito nos fumantes.

Os achados do presente estudo sobre a distribuição do tabagismo segundo sexo e escolaridade concordam com a literatura, apoiando a validade dos resultados. As maiores freqüências de tabagistas têm sido observadas em homens 20 e indivíduos com baixa escolaridade 21 . Quanto à idade, os resultados mostraram uma situação complexa e difícil de explicar, caracterizada por alta freqüência de jovens fumantes (18-29 anos), queda abrupta na faixa seguinte, novamente uma elevação na faixa de 40-49 anos, seguida 
Tabela 1

Prevalência de tabagismo em homens e mulheres maiores de 18 anos segundo faixa etária, escolaridade e estado nutricional. Botucatu, São Paulo, Brasil, 2004.

\begin{tabular}{|c|c|c|c|c|c|}
\hline \multirow[t]{2}{*}{ Variáveis } & \multicolumn{2}{|c|}{ Homens } & \multicolumn{2}{|c|}{ Mulheres } & \multirow[t]{2}{*}{ Valor de $p$} \\
\hline & $\mathbf{n}$ & $\%$ & $\mathrm{n}$ & $\%$ & \\
\hline Idade (anos) & & & & & $<0,0001$ \\
\hline $18-29$ & 75 & 45,2 & 47 & 33,3 & \\
\hline $30-39$ & 19 & 11,4 & 22 & 15,6 & \\
\hline $40-49$ & 36 & 21,7 & 46 & 32,6 & \\
\hline $50-59$ & 22 & 13,3 & 16 & 11,3 & \\
\hline $60+$ & 14 & 8,4 & 10 & 7,1 & \\
\hline Escolaridade (anos) & & & & & $<0,001$ \\
\hline $0-8$ & 131 & 31,5 & 107 & 24,0 & \\
\hline $9-11$ & 26 & 15,6 & 26 & 14,3 & \\
\hline$\geq 12$ & 8 & 8,2 & 8 & 8,0 & \\
\hline Índice de massa corporal (kg/m²) & & & & & $<0,0001$ \\
\hline$<25$ (sem excesso de peso) & 107 & 33,2 & 79 & 22,6 & \\
\hline $25,0-29,9$ (sobrepeso) & 54 & 24,0 & 18 & 10,1 & \\
\hline$\geq 30,0$ (obesidade) & 3 & 3,4 & 20 & 18,5 & \\
\hline
\end{tabular}

Tabela 2

Prevalência de tabagismo e de tabagismo associado a outros fatores de risco de doenças e agravos crônicos não transmissíveis em adultos. Botucatu, São Paulo, Brasil, 2004.

\begin{tabular}{|c|c|c|c|c|}
\hline \multirow[t]{2}{*}{ Fatores de risco } & \multicolumn{2}{|c|}{ Homens } & \multicolumn{2}{|c|}{ Mulheres } \\
\hline & $\mathbf{n}$ & $\%$ & $\mathbf{n}$ & $\%$ \\
\hline 1. Tabagismo & 166 & 24,3 & 141 & 19,4 \\
\hline 2. Tabagismo + sedentarismo & 95 & 13,9 & 103 & 14,2 \\
\hline 3. Tabagismo + baixo consumo de frutas & 88 & 12,9 & 90 & 12,3 \\
\hline 4. Tabagismo + excesso de peso & 56 & 8,6 & 40 & 5,5 \\
\hline 5. Tabagismo + baixo consumo de saladas & 40 & 5,8 & 37 & 5,1 \\
\hline 6. Tabagismo + nenhum alimento regulador & 29 & 4,2 & 24 & 3,3 \\
\hline 7. Tabagismo + consumo excessivo de álcool & 24 & 3,5 & 0 & 0,0 \\
\hline 10. Tabagismo + sedentarismo + consumo excessivo de álcool & 9 & 1,3 & 0 & 0,0 \\
\hline
\end{tabular}

de queda contínua com o envelhecimento. É possível que muitos jovens que se declararam tabagistas estivessem experimentando o tabaco; nas faixas etárias mais velhas, o declínio da freqüência de tabagistas é esperado, uma vez que aumenta a cessação do hábito de fumar por conta da preocupação com a saúde ou da presença de doenças 22 .

Estudos internacionais 23,24,25 observaram significativa relação inversa entre uso regular de tabaco e peso corporal, que tende a ser mais baixo entre fumantes quando comparados a não fu- mantes. A ação do fumo no peso corporal parece ser mediada pela nicotina, que induz à supressão do apetite, provavelmente pelo aumento dos neurotransmissores anorexígenos dopamina e serotonina 26 , intensificada pelo possível aumento sérico de leptina nos fumantes 27 . Outra ação da nicotina é o aumento da atividade adrenérgica que induz a termogênese e a conseqüente redução do peso corporal 25,28. No presente estudo a proporção de tabagistas foi mais baixa nos homens obesos, mas não nas mulheres. Um IMC médio maior em mulheres fumantes quando 
Prevalência de tabagismo associado a outros fatores de risco de doenças e agravos crônicos não transmissíveis segundo escolaridade em adultos. Botucatu, São Paulo, Brasil, 2004.

\begin{tabular}{|c|c|c|c|c|c|c|c|}
\hline \multirow[t]{3}{*}{ Fatores de risco/sexo } & \multicolumn{7}{|c|}{ Escolaridade (anos) } \\
\hline & \multicolumn{2}{|c|}{$0-8$} & \multicolumn{2}{|c|}{$9-11$} & \multicolumn{2}{|c|}{$\geq 12$} & \multirow[t]{2}{*}{ Valor de $p$} \\
\hline & $\mathbf{n}$ & $\%$ & n & $\%$ & $\mathbf{n}$ & $\%$ & \\
\hline \multicolumn{8}{|c|}{ Tabagismo + álcool em excesso } \\
\hline Homens & 21 & 5,0 & 3 & 1,8 & 0 & 0,0 & $<0,0001$ \\
\hline Mulheres & - & - & - & - & - & - & - \\
\hline \multicolumn{8}{|c|}{ Tabagismo + sedentarismo } \\
\hline Homens & 78 & 18,8 & 13 & 7,7 & 4 & 4,1 & $<0,0001$ \\
\hline Mulheres & 87 & 19,5 & 13 & 7,1 & 4 & 4,0 & $<0,0001$ \\
\hline \multicolumn{8}{|c|}{ Tabagismo + excesso de peso } \\
\hline Homens & 40 & 10,2 & 12 & 7,4 & 4 & 4,1 & $<0,0001$ \\
\hline Mulheres & 27 & 7,1 & 14 & 6,2 & 2 & 2,0 & $<0,0001$ \\
\hline
\end{tabular}

comparadas com não fumantes já foi observado no Brasil 29. Uma possível explicação para este resultado poderia ser o fato de as mulheres obesas estarem fumando para emagrecer ou controlar o ganho de peso. Dada a natureza transversal e quantitativa deste estudo, não é possível testar esta hipótese, cabendo a novos estudos com desenho longitudinal e a estudos qualitativos semelhante tarefa.

O principal foco do presente estudo foi a quantificação da simultaneidade de outros fatores de risco junto ao tabagismo. Considerando o tabagismo comum para todas as associações de fatores pesquisadas, sua ocorrência com o sedentarismo foi a mais comum, para ambos os sexos, seguida de tabagismo e baixo consumo de frutas e tabagismo e baixo consumo de legumes. Sedentarismo, baixo consumo de frutas e de legumes são comportamentos de risco de DANT muito freqüentes na população em geral $15,16,21,30$. Assim, não foi surpresa que tais fatores apareceram como os mais comuns em associação ao tabagismo. Todavia, como a freqüência de sedentarismo nos tabagistas foi maior do que na população em geral do município ( $56 \%$ vs. $43,1 \%$ em homens e $62 \%$ vs. 58,2\% em mulheres), há indícios de um efeito de aglomeração entre os dois fatores, particularmente evidente nos homens. O mesmo efeito parece existir entre tabagismo e baixo consumo de frutas, nas mulheres $(62,7 \%$ nas tabagistas $v s .47,7 \%$ na população) e entre tabagismo e consumo excessivo de álcool, nos homens $(18,1 \%$ nos tabagistas $v$. $6,8 \%$ na população em geral).

Estimativas da existência de aglomeração de fatores comportamentais de risco de DANT, reali- zadas por estudos internacionais 4,5,9,10, levaram ao reconhecimento de que muitos destes estão inter-relacionados e participam como intermediários em uma cadeia causal. Como conseqüência, uma efetiva prevenção só seria alcançada com a melhoria da ocorrência concomitante dos vários fatores de risco comportamentais modificáveis associados às DANT, nos indivíduos e nas populações.

Há evidências consistentes de que, isoladamente, todos os fatores comportamentais pesquisados e freqüentes na população adulta estudada aumentam o risco de doenças e de morte. Evidências epidemiológicas mostram associação inversa entre consumo adequado de frutas e vegetais e risco de doenças cardiovasculares 31 . Deve-se lembrar que no presente estudo a segunda mais freqüente associação de fatores foi tabagismo e baixo consumo de frutas $(12,9 \%$ nos homens e $12,3 \%$ nas mulheres).

Os hábitos alimentares do fumante se devem à redução do paladar e do olfato, conseqüente à ação da nicotina no sistema nervoso central, provavelmente pelo aumento dos neurotransmissores anorexígenos dopamina e serotonina 25,26 , fazendo-os consumir menos vegetais e frutas, e, muitas vezes, sobrecarregar nos temperos e no sal. A ocorrência de vários hábitos alimentares inadequados em tabagistas é muito comum: alto consumo de café, de bebidas alcoólicas e de guloseimas, estas últimas para tentar eliminar o odor da fumaça.

Consumo excessivo de álcool em combinação com outros fatores comportamentais de risco 4,5,9,32, inclusive com tabagismo, tem sido detectado. Estudando apenas adultos jovens, Guti- 
érrez et al. 5 observaram a associação de tabagismo e consumo de álcool em $10 \%$ da população masculina de Santa Fé, na Colômbia, fatores não observados em conjunto nas mulheres. A freqüência em Botucatu desses dois fatores juntos nos homens acima de 18 anos foi menor (3,5\%) e também não foi observada nas mulheres.

Vários estudos de agregação de fatores encontraram associação inversa entre sua ocorrência e aumento da escolaridade 30,32,33. Em Botucatu, quanto maior o nível de escolaridade, menor o consumo de tabaco associado ao sedentarismo e menor a freqüência de tabagismo e excesso de peso, em ambos os sexos, sugerindo que mais conhecimento influencia positivamente na adoção conjunta de diversos hábitos saudáveis. A escolaridade, variável consistentemente associada com melhores indicadores de saúde, desponta como fator de proteção contra a ocorrência simultânea de comportamentos não saudáveis, apoiando investimentos no aumento da escolaridade de crianças e jovens como promissora política pública de promoção da saúde e redução de DANT. Por outro lado, os indivíduos menos escolarizados devem ser alvo preferencial de ações educativas de prevenção e controle de hábitos não saudáveis.

\section{Resumo}

Foram entrevistados via ligação telefônica 1.410 indivíduos, amostra aleatória e representativa da população acima de 18 anos residente em domicílios conectados à rede de telefonia fixa. A prevalência de tabagismo foi de 21,8\%, maior em homens (25\%) e em indivíduos na faixa entre 18 e 29 anos. Tabagismo e sedentarismo juntos ocorrem em 13,9\% dos homens e 14,2\% das mulheres; tabagismo e baixo consumo de frutas em 12,9\% dos homens e 12,3\% das mulheres; e tabagismo e baixo consumo de legumes em 5,8\% dos homens e 5,1\% das mulheres. A associação de tabagismo e consumo excessivo de álcool foi observada apenas nos homens (em $3,5 \%$ deles) e, da mesma forma que verificada para tabagismo isoladamente, sua ocorrência concomitante a outros fatores comportamentais de risco de doenças e agravos crônicos não transmissiveis (DANT) associouse inversamente à escolaridade. Os dados apontam indícios de efeito de aglomeração entre tabagismo e sedentarismo, tabagismo e álcool em excesso, tabagismo e dieta inadequada, justificando intervenções focadas na prevenção e redução concomitante dos principais fatores comportamentais de risco de DANT.

Tabagismo; Estilo de Vida Sedentário; Estilo de Vida; Hábitos Alimentares
Como as DANT compartilham vários fatores de risco, e como tais fatores não ocorrem isoladamente nos indivíduos, o enfoque da OMS, que propõe uma abordagem integrada de prevenção e controle, em todas as idades, baseada na redução concomitante dos principais fatores comportamentais (fumo, sedentarismo, álcool em excesso, dieta inadequada e obesidade), deve ser adotado 34 .

\section{Conclusão}

A prevalência de tabagismo na população de Botucatu foi de $21,8 \%$, sendo maior nos homens, na população mais jovem e com menor nível de escolaridade. Combinações de tabagismo e outros fatores comportamentais de risco de DANT ocorreram em frequências expressivas, sendo tabagismo associado a sedentarismo a mais freqüente $(13,9 \%$ nos homens e $14,2 \%$ nas mulheres), seguida de tabagismo e baixo consumo de frutas e hortaliças. Em homens, 3,5\% são tabagistas e apresentam consumo excessivo de álcool. A ocorrência desses fatores nos tabagistas foi maior do que na população em geral, indicando um possível efeito de aglomeração de fatores.

\section{Colaboradores}

S. J. P. Berto realizou a análise dos resultados e elaborou o artigo. M. A. B. L. Carvalhaes elaborou do projeto inicial, executou a coleta de dados e elaborou o artigo. E. C. Moura elaborou o projeto inicial, executou o projeto, analisou os resultados e revisou o artigo. 


\section{Referências}

1. Organización Mundial de la Salud. Vigilancia de los factores de riego relacionados con enfermedades no transmisibles: estado actual de la información en el mundo. Genebra: Organización Mundial de la Salud; 2003. (Informe SuRF, 1).

2. Rosini N, Machado MJ, Xavier HT. Estudo de prevalência e multiplicidade de fatores de risco cardiovascular em hipertensos do município de Brusque, SC. Arq Bras Cardiol 2006; 86:219-22.

3. Kelishadi R, Sadri G, Tavasoli AA, Kahbazi M, Roohafza HR, Sadeghi M, et al. A prevalência cumulativa de fatores de risco para doença cardiovascular em adolescentes iranianos - IHHP-HHPC. J Pediatr 2005; 81:447-53.

4. Galán I, Rodriguez-Artalejo F, Tobías A, Díez-Gañán L, Gandarilhas A, Zorrilla B. Agregación de factores de riesgo ligados al comportamiento y su relación con la salud subjetiva. Gac Sanit 2005; 19:1-9.

5. Gómez-Gutiérrez LF, Lucumí-Cuesta DI, GirónVargas SL, Espinosa-García G. Conglomeración de factores de riesgo de comportamiento asociados a enfermedades crónicas en adultos jóvenes de dos localidades de Bogotá, Colombia: importancia de las diferencias de género. Rev Esp Salud Pública 2004; 78:493-504.

6. Meng L, Maskarinec G, Lee J, Kolonel LN. Lifestyle factors and chronic diseases: application of a composite risk index. Prev Med 1999; 29:296-304.

7. Yusuf HR, Giles WH, Croft JB, Anda RF, Casper ML. Impact of multiple risk factor profiles on determining cardiovascular disease risk. Prev Med 1998; 27:1-9.

8. Li FX, Robson PJ, Chen Y, Qiu Z, Siou GL, Bryant HF. Prevalence, trend, and sociodemographic as sociation of five modifiable lifestyle risk factors for cancer in Alberta and Canada. Cancer Causes Control 2009; 20:395-407.

9. Poortinga W. The prevalence and clustering of four lifestyle risk factors in an English adult population. Prev Med 2007; 427:124-8.

10. Schit AJ, Loon AJMV, Tizhuis M, Ocke MC. Clustering of lifestyle risk factors in a general adult population. Prev Med 2002; 35:219-24.

11. World Health Organization. WHO report on the global tobacco epidemic, 2008: the MPOWER package. Geneva: World Health Organization; 2008.

12. Capilheira MF, Santos IS, Azevedo Jr. MR, Reichert FF. Risk factors for chronic non-communicable diseases and the CARMEN Initiative: a populationbased study in the South of Brazil. Cad Saúde Pública 2008; 24:2767-74.

13. Lessa I, Araújo MJ, Magalhães L, Almeida Filho N, Aquino E, Costa MCR. Simultaneidade de fatores de risco cardiovascular modificáveis na população adulta de Salvador (BA), Brasil. Rev Panam Salud Pública 2004; 16:131-7.

14. Secretaria de Gestão Estratégica e Participativa, Secretaria de Vigilância em Saúde, Ministério da Saúde. Vigitel Brasil 2008: vigilância de fatores de risco e proteção para doenças crônicas por inquérito telefônico. Brasília: Ministério da Saúde; 2009.
15. Carvalhaes MABL, Moura EC, Monteiro CA. Prevalência de fatores de risco para doenças crônicas: inquérito populacional mediante entrevistas telefônicas em Botucatu, São Paulo, 2004. Rev Bras Epidemiol 2008; 11:14-23.

16. Monteiro CA, Moura EC, Jaime PC, Lucca A, Florindo AA, Figueiredo ICR, et al. Monitoramento de fatores de risco para doenças crônicas por entrevistas telefônicas. Rev Saúde Pública 2005; 39:47-57.

17. Instituto Brasileiro de Geografia e Estatística. Censo demográfico 2000 [CD-ROM]. Rio de Janeiro: Instituto Brasileiro de Geografia e Estatística; 2003.

18. Monteiro CA, Florindo AA, Claro RM, Moura EC. Validade de indicadores de atividade física e sedentarismo obtidos por inquérito telefônico. Rev Saúde Pública 2008; 42:575-81.

19. Monteiro CA, Moura EC, Jaime PC, Claro RM. Validade de indicadores do consumo de alimentos e bebidas obtidos por inquérito telefônico. Rev Saúde Pública 2008; 42:582-9.

20. Marcopito LF, Rodrigues SSF, Pacheco MA, Shirassu MM, Goldfeder AJ, Moraes MA. Prevalência de alguns fatores de risco para doenças crônicas na cidade de São Paulo. Rev Saúde Pública 2005; 39:738-45

21. Peixoto MRG, Monego ET, Alexandre VP, Souza RGM, Moura EC. Monitoramento por entrevistas telefônicas de fatores de risco para doenças crônicas: experiência de Goiânia, Goiás, Brasil. Cad Saúde Pública 2008; 24:1323-33.

22. Peixoto SV, Firmo JOA, Lima-Costa MF. Fatores associados ao índice de cessação do hábito de fumar em duas diferentes populações adultas (Projetos Bambuí e Belo Horizonte). Cad Saúde Pública 2007; 23:1319-28.

23. Albanês D, Jones DY, Micizzi MS, Mattson ME. Associations between smoking and body weight in the US population: analysis of NHANES II. Am J Public Health 1987; 77:439-44.

24. Klesges RC, Ward KD, Ray JW, Cutter G, Jacobs Jr. DR, Wagenknechit LE. The prospective relationships between smoking and weight in a young, biracial cohort: the Coronary Arterial Risk Development in Young Adults Study. J Consult Clin Psychol 1998; 66:987-93.

25. Chatkin R, Chatkin JM. Tabagismo e variação ponderal: a fisiologia e genética podem explicar esta associação? J Bras Pneumol 2007; 33:712-9.

26. Klein LC, Corwin EJ, Ceballos RM. Leptin, hunger, and body weight: influence of gender, tobacco smoking, and smoking abstinence. Addict Behav 2004; 29:921-7.

27. Nicklas BJ, Tomoyasu N, Muir J, Goldberg AP. Effects of cigarette smoking and its cessation on body weight and plasma leptin levels. Metabolism 1999; 48:804-8.

28. Pisinger C, Jorgensen T. Waist circumference and weight following smoking cessation in a general population: the Inter99 study. Prev Med 2007; 44:290-9. 
29. Peixoto MRG, Benicio MHA, Jardim PCBV. The relationship between body mass index and lifestyle in a Brazilian adult population: a cross-sectional survey. Cad Saúde Pública 2007; 23:2694-704.

30. Van Eyken EBBD, Moraes CL. Prevalência de fatores de risco para doenças cardiovasculares entre homens de uma população urbana do Sudeste do Brasil. Cad Saúde Pública 2009; 25:111-23.

31. Barreto SM, Pinheiro ARO, Sichieri R, Monteiro CA, Batista Filho M, Schimidt MI, et al. Análise da estratégia global para a alimentação saudável, atividade física e saúde da Organização Mundial da Saúde. Epidemiol Serv Saúde 2005; 14:41-68.
32. Pereira JC, Barreto SM, Passos VMA. O perfil de saúde cardiovascular dos idosos brasileiros precisa melhorar: estudo de base populacional. Arq Bras Cardiol 2008; 91:1-10.

33. Peixoto SV, Firmo JOA, Lima-Costa MF. Factors associated to smoking habit among older adults (The Bambuí Health and Aging Study). Rev Saúde Pública 2005; 39:745-53.

34. Organização Pan-Americana da Saúde. Doenças crônico-degenerativas e obesidade: estratégia mundial sobre alimentação saudável, atividade física e saúde. Brasília: Ministério da Saúde; 2003.

Recebido em 13/Ago/2009

Versão final reapresentada em 30/Mar/2010

Aprovado em 10/Jun/2010 